\title{
Correction to: Abstracts of the 15th International Congress of the European Geriatric Medicine Society
}

\author{
Editorial Office of European Geriatric Medicine ${ }^{1}$
}

Published online: 11 February 2020

(c) European Geriatric Medicine Society 2020

\section{Correction to: Eur Geriatr Med (2019) 10 (Suppl 1):S1-S325 https://doi.org/10.1007/s41999-019-00221-0}

The Editorial Office of EGM has been informed that the abstract P-706 is not correct.

The correct version of the abstract P-706 is the following:

The assessment of clinical applicability of polish version of SARC-F as a sarcopenia case-finding tool

Karolina Piotrowicz ${ }^{1}$, Anna Głuszewska ${ }^{1}$, Joanna Czesak ${ }^{2}$, Małgorzata Fedyk-Łukasik ${ }^{1}$, Anna Skalska ${ }^{1}$, Barbara Gryglewska ${ }^{1}$, Tomasz Grodzicki ${ }^{1}$, Jerzy Gąsowski ${ }^{1}$

${ }^{1}$ Department of Internal Medicine and Gerontology, Medical College, Jagiellonian University, Kraków, Poland

${ }^{2}$ Department of Clinical Rehabilitation, University School of Physical Education, Kraków, Poland

Introduction: Sarcopenia is a prevalent yet potentially reversible syndrome, hence the need for a simple screening method. SARC-F questionnaire for case finding is a five-item instrument introduced into the European Working Group on sarcopenia in Older People 2 (EWGSOP2) algorithm for diagnosis of sarcopenia.

The original article can be found online at https://doi.org/10.1007/ s41999-019-00221-0.

\section{Editorial Office of European Geriatric Medicine}

https://www.springer.com/journal/41999
Methods: We used data from a cross-sectional study aimed at cultural and clinical validation of the Polish translation of SARC-F in community-dwelling subjects $\geq 65$ years. Diagnosis of sarcopenia was based on the 2018 EWGSOP 2 consensus. Hand grip and 4-m gait speed were measured, and the Polish version of SARC-F was administered.

Results: The mean (SD) age of 73 participants (21.9\% men) was 77.8 (7.3) years. Seventeen participants (23.3\%) fulfilled the EWGSOP2 criteria of sarcopenia, and $9(12.3 \%)$ criteria for severe sarcopenia. Fourteen (19.2\%) participants fulfilled the SARC-F criteria for clinical suspicion of sarcopenia. The Cronbach's alpha coefficient for internal consistency of the SARC-F test was 0.84. With EWGSOP2 sarcopenia as gold standard, the sensitivity of SARC-F was 35.3\% (95\% CI $14.2-61.7, p=0.33)$ and specificity was $85.7 \%$ (95\% CI 73.8-93.6, $p<0.0001)$. The corresponding PPV and NPV were $42.9 \%(p=0.79)$ and $81.4 \%(p<0.0001)$, respectively. The probability of false positive result was $14.3 \%$ (95\% CI $6.4-26.2, p<0.0001)$ and the probability of false negative result was $64.7 \%$ (95\% CI 38.3-85.8, $p=0.33$ ). Overall, the predictive power of SARC-F was low (the ROC 0.64).

Key conclusions: SARC-F is currently recommended for sarcopenia case finding in the general population of older adults. However, due to the low sensitivity, despite high specificity its further development seems to be needed.

Publisher's Note Springer Nature remains neutral with regard to jurisdictional claims in published maps and institutional affiliations. 